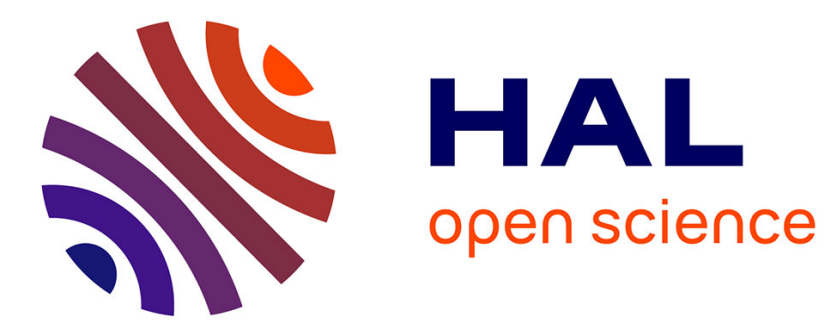

\title{
Modéliser le dernier maximum glaciaire et l'Holocène moyen
}

Pascale Braconnot

\section{To cite this version:}

Pascale Braconnot. Modéliser le dernier maximum glaciaire et l'Holocène moyen. Comptes Rendus Géoscience, 2004, 336, pp.711-719. 10.1016/j.crte.2003.12.023 . hal-03023563

\section{HAL Id: hal-03023563 https://hal.science/hal-03023563}

Submitted on 25 Nov 2020

HAL is a multi-disciplinary open access archive for the deposit and dissemination of scientific research documents, whether they are published or not. The documents may come from teaching and research institutions in France or abroad, or from public or private research centers.
L'archive ouverte pluridisciplinaire HAL, est destinée au dépôt et à la diffusion de documents scientifiques de niveau recherche, publiés ou non, émanant des établissements d'enseignement et de recherche français ou étrangers, des laboratoires publics ou privés. 


\title{
Modéliser le dernier maximum glaciaire et l'Holocène moyen
}

\author{
Pascale Braconnot \\ Laboratoire des sciences du climat et de l'environnement, IPSL/LSCE, unité mixte CEA-CNRS, Orme des Merisiers, \\ bât. 701, 91991 Gif-sur-Yvette cedex, France \\ Accepté le 10 décembre 2003
}

Rédigé à l'invitation du Comité éditorial

\begin{abstract}
Résumé
Les variations climatiques passées représentent un véritable test de notre compréhension du système climatique et des principales interactions entre les différents milieux qui le composent. Cet article présente l'évolution de la modélisation tridimensionnelle pour les périodes du dernier maximum glaciaire et de l'Holocène moyen. À partir des simulations du projet international PMIP (Paleoclimat Modeling Intercomparison Project) et de simulations où l'atmosphère est couplée à un modèle d'océan ou de végétation, nous montrons comment ces modèles complexes nous permettent d'aborder de nouvelles questions Les périodes choisies permettent d'étudier la réponse du système climatique à un forçage annuel et un forçage saisonnier. Pour citer cet article $:$ P. Braconnot, C. R. Geoscience 336 (2004).
\end{abstract}

(C) 2004 Académie des sciences. Publié par Elsevier SAS. Tous droits réservés.

\section{Abstract}

Modeling the Last Glacial Maximum and Mid-Holocene. Past climate conditions represent a many faceted challenge for our understanding of the natural variability of the climate system and of the major interactions between its various components. This paper presents a short review of the evolution of past climate simulations using three-dimensional models for the Last Glacial Maximum and the mid-Holocene climates. Its shows how the increase in model complexity allows to test new ideas and mechanisms, using results from the Paleoclimate Modeling Intercomparison Project (PMIP) and from simulations where the atmospheric model is coupled to an ocean or a vegetation model. The two periods are respectively representative of the response of the climate system to an annual mean forcing and a seasonal forcing. To cite this article: P. Braconnot, C. R. Geoscience 336 (2004).

๑ 2004 Académie des sciences. Publié par Elsevier SAS. Tous droits réservés.

Mots-clés : modélisation des paléoclimats ; couplage océan-atmosphère-végétation ; rétroactions climatiques ; évaluation des modèles

Keywords: paleoclimate modeling; coupling between ocean; atmosphere and vegetation; climate feedbacks; model testing

\section{Introduction}

Le climat de la terre est le résultat de multiples interactions entre des processus faisant intervenir l'at-

Adresse e-mail : pasb@1sce.saclay.cea.fr (P. Braconnot). mosphère, l'océan et les surfaces continentales. Les projections climatiques pour le prochain siècle reposent sur notre capacité à modéliser ces phénomènes complexes. Bien que les résultats des modèles de climat soient de plus en plus fiables, l'augmentation de température moyenne simulée à l'horizon de 2100 se 
situe dans une fourchette de 1,4 à $5,8^{\circ} \mathrm{C}$ [14]. Une partie des incertitudes liées à ces projections climatiques provient de la difficulté de représenter certains processus physiques ou de l'omission d'interactions intervenant au sein du système climatique.

Comprendre comment le climat a varié dans le passé représente un véritable test de notre compréhension du système climatique et de notre capacité à le modéliser. Le projet international PMIP [15] a été créé en 1991 pour faciliter les analyses systématiques des modèles de climat et évaluer la capacité de ces modèles à reproduire un climat différent de l'actuel. PMIP a aussi joué un rôle important dans les synthèses de données permettant d'évaluer les résultats des modèles au regard du climat passé. La première phase du projet a considéré deux périodes représentant des conditions extrêmes dans le passé et pour lesquelles il existe de nombreuses données permettant de les caractériser. Le dernier maximum glaciaire (DMG), il y a 21000 ans, est une période froide, caractérisée par d'importantes calottes de glace de 2 à $3 \mathrm{~km}$ d'épaisseur sur l'Amérique du Nord et le Nord de l'Europe, ainsi que par une concentration moindre en gaz carbonique dans l'atmosphère. Le climat de l'Holocène moyen, il y a 6000 ans, se caractérise par un contraste saisonnier accru (diminué) du rayonnement solaire incident au sommet de l'atmosphère dans l'hémisphère nord (sud) d'environ $5 \%$. Cette modification du forçage solaire s'accompagne d'une amplification du cycle hydrologique dans les régions tropicales.

L'étude des variations climatiques passées fait appel, tout comme l'étude du climat actuel, à toute une hiérarchie de modèles, allant des modèles conceptuels aux modèles de circulation générale, auxquels s'intéresse cet article, qui sont les plus complexes et les plus coûteux en temps de calcul. Ces derniers sont basés sur les équations de la dynamique des fluides géophysiques, auxquelles est adjoint un ensemble de paramétrisations physiques, permettant de représenter les phénomènes physiques d'échelle inférieure à la maille. Ainsi, même si certaines paramétrisations sont relativement simples, les cycles de l'eau et de l'énergie sont explicitement représentés dans ces modèles. Pour représenter un climat passé, la solution consiste à se placer autour de la période que l'on souhaite simuler et à réaliser une simulation suffisamment longue pour être représentative de la période en question et le cas échéant de sa variabilité.

Deux expériences PMIP ont ainsi été définies pour le dernier maximum glaciaire. La première expérience considère les températures de surface de l'océan (TSOs) prescrites suivant les reconstructions CLIMAP [6]. Cette expérience permet de réaliser la simulation la plus réaliste possible, mais les résultats sont dépendants des larges incertitudes attachées à la reconstruction des TSOs. Pour la deuxième expérience, le modèle d'atmosphère est couplé à un modèle simplifié de couche de mélange océanique, qui calcule la TSO à partir des flux de chaleur à l'interface air-mer et du transport de chaleur océanique, imposé à partir de la simulation de contrôle ou d'estimations obtenues pour le climat actuel. Cette configuration de modèle est largement utilisée pour les simulations permettant d'évaluer la sensibilité climatique à un doublement de $\mathrm{CO}_{2}$. Néanmoins, l'hypothèse faite sur le transport de chaleur océanique n'est pas réaliste. Les données des carottes océaniques suggèrent une réduction de la circulation thermohaline à cette époque [8]. Pour toutes les expériences, les calottes de glace sont issues de Peltier [24]. La concentration atmosphérique en $\mathrm{CO}_{2}$ provient des résultats des carottes Antarctiques [26] et est fixée à 200 ppm.

La différence d'insolation entre l'Holocène moyen et la période actuelle provient de la configuration orbitale de la Terre dans son mouvement autour du soleil et principalement de la longitude du périhélie. Les autres paramètres orbitaux sont peu différents de l'actuel [1]. La concentration de $\mathrm{CO}_{2}$ pour l'Holocène moyen est fixée à la valeur préindustrielle, 280 ppm. Contrairement au dernier maximum glaciaire, il n'existe pas de reconstruction globale des TSOs pour cette époque. Les données disponibles au début du projet PMIP indiquaient que les différences par rapport à la période actuelle étaient faibles [21,27]. Il était donc raisonnable de considérer les TSOs actuelles comme condition aux limites sur les océans.

Le projet PMIP était limité aux seuls modèles d'atmosphère. Néanmoins, ces dernières années ont vu le développement spectaculaire des modèles couplés océan-atmosphère et atmosphère-végétation. Le rôle du changement de couvert végétal a été le premier analysé. Les études les plus nombreuses concernent l'Holocène moyen [5,7,29]. La prise en compte des changements de circulation océanique a aussi été réa- 
lisée tout d'abord pour l'Holocène moyen [3,11,22, 23 ] et, plus récemment, pour le dernier maximum glaciaire $[12,19,20,28]$. Peu de simulations considèrent les rétroactions simultanées de l'océan et le la végétation [2]. La suite de ce document présente tout d'abord les principaux résultats de PMIP (Section 2). La façon dont la réponse de l'océan ou celle du couvert végétal modifie les résultats et permet d'aborder l'étude du changement de variabilité climatique est ensuite discutée dans les Sections 3 et 4, en se basant sur les résultats de la littérature ou du groupe de travail sur les simulations couplées au moyen Holocène issu de PMIP.

\section{Résultats PMIP pour le dernier maximum glaciaire et l'Holocène moyen}

Le refroidissement moyen simulé pour le DMG est de l'ordre de $4{ }^{\circ} \mathrm{C}$ pour les simulations avec TSOs prescrites et varie de 6 à $2^{\circ} \mathrm{C}$ pour les simulations avec TSOs calculées. Le refroidissement est plus marqué sur les continents que sur les océans, en raison de la plus faible capacité thermique du sol. Il est maximal dans l'hémisphère nord au-dessus des calottes de glace. La modification associée des gradients méridiens de température dans l'océan Atlantique nord favorise un déplacement vers l'est du cœur de la zone de passage dépressionnaire. Cette modification de la baroclinicité de l'écoulement s'ajoute à l'effet orographique induit par la présence des calottes de glace [17]. Aux moyennes et hautes latitudes, les comparaisons avec les reconstructions climatiques issues des données de pollen indiquent un relativement bon accord entre modèles et données sur l'Eurasie, mais un désaccord important sur l'Europe [18]. De nombreuses questions restent en suspens pour expliquer ce désaccord. Serait-il dû au relief européen mal représenté dans les modèles, aux températures océaniques mal simulées ou incorrectes dans CLIMAP? À des incertitudes dans les reconstructions elles-mêmes ? Différentes pistes sont explorées actuellement.

Dans les régions tropicales, le refroidissement est plus marqué en été qu'en hiver pour les simulations avec TSO prescrites (Fig. 1(a)). Cette période froide est aussi une période plus aride sur la plupart des continents, comme l'indique la réduction de précipitation (Fig. 1(a)). L'atmosphère ayant une capacité moindre à stocker la vapeur d'eau lorsque la température diminue, le cycle hydrologique est amorti. L'évaporation est également moins efficace sur les océans, excepté sur les poches chaudes très controversées des TSOs CLIMAP, ce qui explique que les modèles avec TSO prescrite ne produisent pratiquement aucun changement de précipitation en moyenne sur l'océan (Fig. 1(a)). La confrontation des résultats à différents indicateurs climatiques [9] indique que le refroidissement tropical et l'aridification produits par ces simulations sont insuffisants. Les résultats des simulations avec TSO calculées simulent sur l'océan une réduction de précipitation équivalente à celle qui est simulée sur le continent (Fig. 1(b)). Le refroidissement continental est également amplifié, mais très dépendant de l'intensité de refroidissement simulé sur l'océan. Le corollaire est que les modèles produisant un refroidissement satisfaisant sur le continent ont des températures de surface océanique trop froides [25].

Le climat de l'Holocène moyen se caractérise par une réponse du système climatique à un forçage saisonnier (insolation) et non, comme pour le dernier maximum glaciaire, à la réponse du système à un forçage annuel moyen (relief, albédo de la glace et $\mathrm{CO}_{2}$ ). En réponse au changement d'insolation, tous les modèles PMIP produisent une amplification du cycle saisonnier des températures continentales dans l'hémisphère nord, qui s'accompagne d'une modification des transports de chaleur et d'eau entre le continent et l'océan. En particulier, la zone de convergence intertropicale est déplacée vers le sud sur les régions océaniques en hiver, ce qui explique qu'il y a plus de précipitation sur l'océan, plus froid, que sur le continent à cette époque (Fig. 1(c)). En été, la zone de convergence intertropicale est déplacée vers le nord et le flux de mousson est accru. Les précipitations sont renforcées sur les continents et diminuées sur l'océan (Fig. 1(c)). Il n'y a donc pas d'adéquation aussi directe qu'au DMG entre les changements de température et de précipitation, car les modifications des gradients terre/océan constituent le mécanisme dominant.

L'amplification de la mousson d'été est significative dans toutes les simulations [16]. Le changement de précipitation de mousson varie de 0 à 


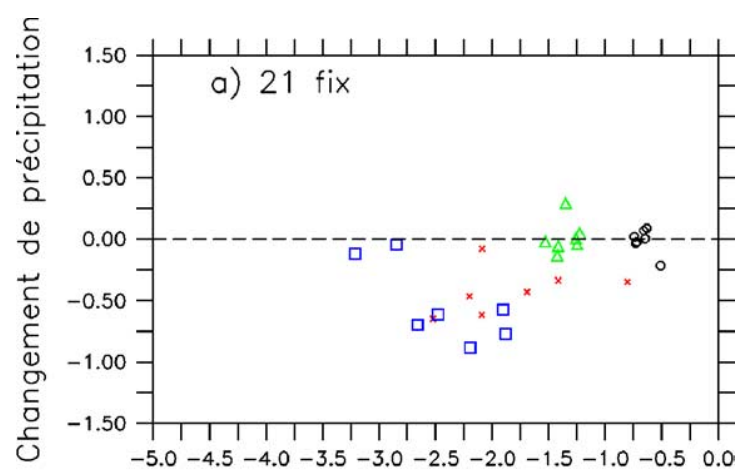

Changement de température

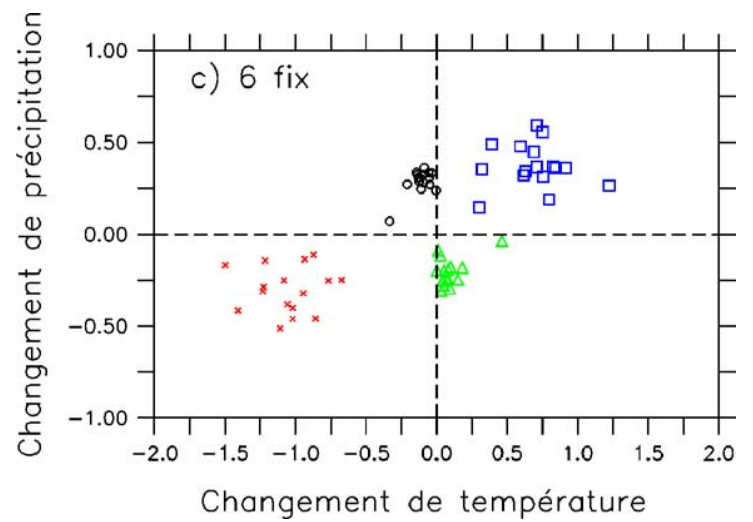

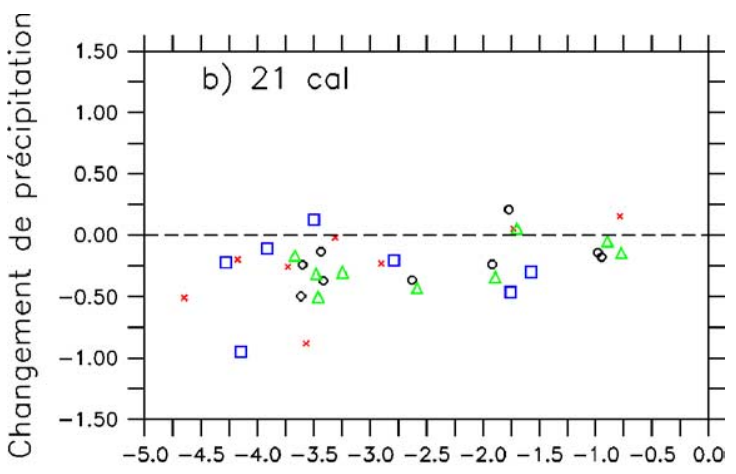

Changement de température

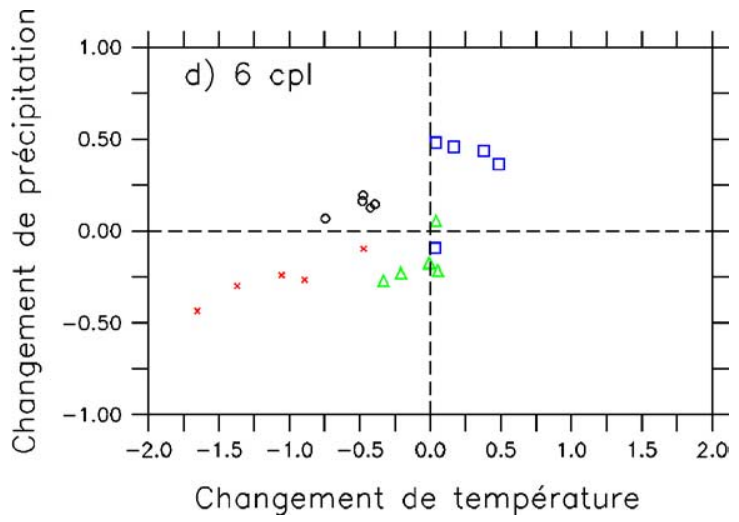

Fig. 1. Changement de précipitation en fonction du changement de température de surface, moyennés entre $20^{\circ} \mathrm{S}$ et $30^{\circ} \mathrm{N}$ sur les océans en hiver (ronds noirs), les continents en hiver (croix rouges), les océans en été (triangles verts), les continents en été (carrés bleus). Chaque point représente le résultat d'un modèle. (a) Sept simulations PMIP du DMG avec TSO prescrites et (b) neuf simulations PMIP du DMG avec TSOs calculées, (c) 16 simulations PMIP de l'Holocène moyen et (d) cinq simulations couplées océan-atmosphère de l'Holocène moyen.

Fig. 1. Change in precipitation as a function of change in surface temperature averaged from $20^{\circ} \mathrm{S}$ to $30^{\circ} \mathrm{N}$ over the ocean in winter (black circles), land in winter (red crosses), ocean in summer (green triangles); land in summer (blue squares). Each model is represented by one point. (a) Seven PMIP simulations of Last Glacial Maximum with fixed SSTs, (b) nine PMIP simulations of Last Glacial Maximum with computed SSTs, (c) 16 PMIP simulations of the mid-Holocene and, (d) five coupled ocean-atmosphere simulations of the mid-Holocene.

2,5 $\mathrm{mm} \mathrm{j}^{-1}$ pour le Nord de 1'Inde et de 0 à $1,5 \mathrm{~mm}^{-1}$ sur l'Afrique de l'Ouest, où il est maximal en août (Fig. 2(a)). En Afrique de l'Ouest, l'intensification des précipitations provient de l'accroissement de l'advection d'air océanique humide et très peu du recyclage local, alors que, pour le climat actuel, le recyclage local intervient pour environ $30 \%$ dans les précipitations de cette région. La gamme des changements de pluie simulés par les différents modèles dans le Nord de l'Inde peut être reliée à la gamme de chauffage continental. Ce dernier dépend des conditions de surface, mais aussi des conditions de nébulosité actuelle et des rétroactions nuageuses. Les caractéristiques des modèles ainsi que la température moyenne de la simulation de contrôle déterminent les différences entre les simulations [4].

Néanmoins, malgré la grande dispersion des résultats, aucun modèle n'est capable de reproduire l'humidification nécessaire pour faire pousser de la végétation de type steppe dans les régions actuellement désertiques $[10,16]$. Ce résultat clef a largement contribué à l'émergence de nombreuses études de sensibilité et de révisions des jeux de données disponibles. 


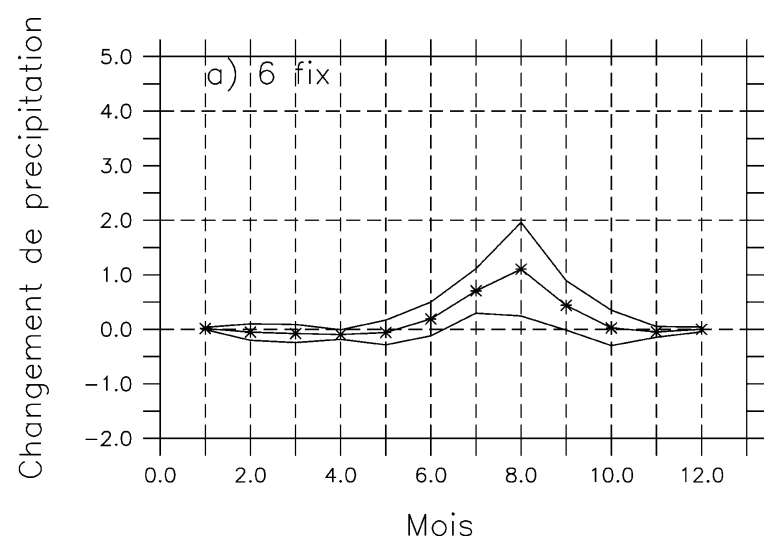

(a)

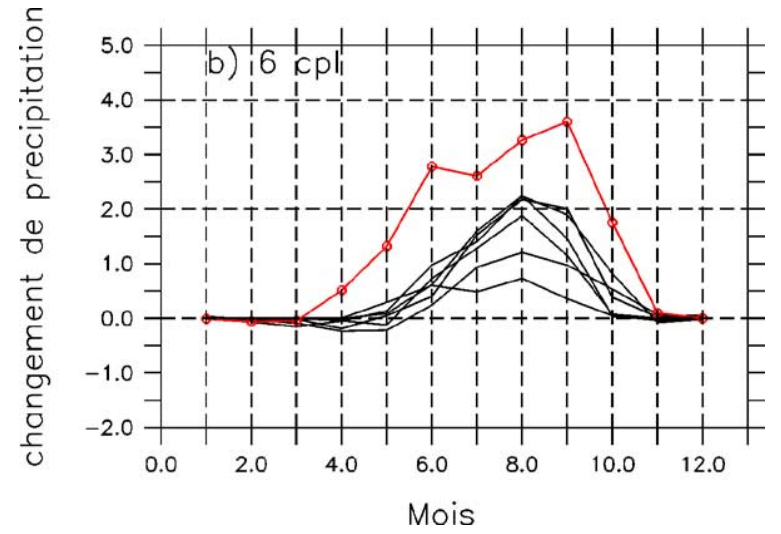

(b)

Fig. 2. Changement de précipitation pour l'Holocène moyen en fonction des mois de l'année, pour une boîte représentative du changement de mousson en Afrique de l'Ouest $\left(17^{\circ} \mathrm{W}-20^{\circ} \mathrm{E}\right.$, $12^{\circ} \mathrm{N}-23^{\circ} \mathrm{N}$ ). (a) Résultats de 16 simulations PMIP : la courbe avec symbole représente la moyenne des simulations et les deux lignes de part et d'autre l'intervalle de confiance à $95 \%$. (b) Résultats de six modèles couplés océan-atmosphère (courbes sans symboles) et de la simulation couplée prenant en compte le couplage avec le changement de végétation (courbe noire avec symboles).

Fig. 2. Change in precipitation for the mid-Holocene, plotted as a function of month for a box located in West Africa $\left(17^{\circ} \mathrm{W}-20^{\circ} \mathrm{E}\right.$, $12^{\circ} \mathrm{N}-23^{\circ} \mathrm{N}$ ). (a) Results of 16 PMIP simulations; the middle curve represent the mean of all simulations and the two extreme lines the $95 \%$ confidence interval. (b) Results of six coupled simulations (black curves) and of a coupled simulation that takes into account changes in the vegetation.

\section{Rétroactions liées à l'océan et à la végétation}

Plusieurs groupes ont maintenant effectué des simulations couplées océan-atmosphère pour le DMG.
Ces simulations ne sont globalement pas en meilleur accord avec les données que les simulations PMIP. Elles n'ont pas toutes suivi le même protocole expérimental. La difficulté majeure vient du temps d'ajustement long (1000 ans) de la circulation océanique. Différentes méthodes d'accélération ont été utilisées pour mettre l'océan dans un mode froid et il n'est pas exclu que ces ajustements biaisent en partie la réponse du système. Le refroidissement obtenu est du même ordre de grandeur que celui obtenu par l'ensemble des modèles PMIP, sauf pour un modèle qui montre un refroidissement de $10^{\circ} \mathrm{C}$ pour la température globale et $6^{\circ} \mathrm{C}$ pour l'océan de surface [19]. Les résultats lèvent de nombreuses questions sur le rôle climatique de la circulation thermohaline de l'océan, la façon dont elle a varié dans le passé et sa représentation dans les modèles de climat. Les études avec des modèles de complexité intermédiaires montrent aussi qu'en période froide du type glaciaire, la circulation thermohaline de l'océan est proche d'un seuil, qui peut la faire basculer dans un état fortement amorti (cf. article de D. Paillard, dans ce numéro). Toutes les simulations couplées suggèrent une migration vers le sud des régions de formation de l'eau profonde nord-atlantique (NADW). Deux des quatre simulations couplées glaciaires présentent un renforcement de la circulation thermohaline dans l'océan Atlantique nord, qui se caractérise par une augmentation du flux de masse dans l'Atlantique nord de plus de 10Sv [12,19]. Les résultats des autres simulations sont en meilleur accord avec les données qui indiquent une ventilation moins importante des eaux de fond et un ralentissement de la circulation océanique (cf. article de J.-C. Duplessy dans ce numéro). La plupart des simulations renforcent la formation d'eau antarctique de fond. Les analyses de Shin et al. [28] montrent que le mécanisme dominant dans l'océan Austral vient d'une augmentation de la densité de surface via l'accroissement de salinité résultant du rejet de sel qui accompagne la formation de la glace de mer.

Les comparaisons des résultats entre un modèle couplé océan-atmosphère à ceux obtenus avec le modèle d'atmosphère couplé à une simple couche de mélange ne permettent pas d'évaluer le rôle de l'océan dans le changement de température global $[13,19]$. Néanmoins, les deux études témoignent de différences de comportement liées au changement de circulation océanique dans les régions de formation 
d'eau profonde et les régions tropicales, dont les processus ne sont pas représentés dans les modèles de couche de mélange.

À l'Holocène moyen, les modèles couplés océanatmosphère ne produisent pas de modification notoire de la circulation thermohaline. La réponse de l'océan agit principalement sur une modification des caractéristiques du cycle saisonnier. En particulier, l'océan transporte moins de chaleur entre l'équateur et les pôles durant l'hiver boréal et participe à l'évacuation du surplus de chaleur de l'hémisphère nord vers l'hémisphère sud pendant l'été [3]. L'océan a une inertie thermique plus importante que le continent. Il répond avec un retard de 1 à 3 mois, suivant les régions, au forçage d'insolation. L'océan tropical est ainsi plus froid qu'à l'actuel au démarrage de la mousson d'été, ce qui renforce l'advection du flux de mousson et permet à la mousson africaine d'être plus intense au mois de juin comparé aux simulations PMIP (Fig. 2(b)). En fin de saison, le réchauffement tardif de l'océan dans l'hémisphère nord favorise un positionnement plus au nord qu'à l'Actuel de la zone de convergence intertropicale sur l'océan Atlantique. L'advection d'humidité associée favorise le maintien de la mousson en Afrique de l'Ouest et l'allongement de la saison de mousson avec des précipitations plus intenses au mois de septembre (Fig. 2(b)).

Les changements de couvert végétal ont également un impact important sur l'amplitude du changement climatique. Au dernier maximum glaciaire, l'extension de la toundra en Asie renforce le refroidissement dans ces régions, alors que le remplacement de forêts par la savane contribue au réchauffement des régions tropicales [31]. Cet effet est cependant moins important que les changements de circulation océanique et la réduction des gaz à effet de serre. A parti d'une simulation couplée glaciaire ne prenant en compte que la réduction des gaz à effet de serre, Shin et al. [28] estiment en effet que cette réduction représente environ la moitié du refroidissement au dernier maximum glaciaire dans les régions tropicales, confortant les résultats d'études antérieures.

De nombreux travaux ont également montré l'impact de la végétation sur les simulations de l'Holocène moyen. Nous illustrons ci-dessous l'effet de la végétation à partir de résultats obtenus en couplant de façon asynchrone un modèle couplé océan-atmosphère et un modèle de biome [2]. Changer la végétation revient à superposer au forçage saisonnier de l'insolation un forçage annuel en surface, lié à l'albédo, à la longueur de rugosité et au profil racinaire de la végétation. Dans les moyennes et hautes latitudes de l'hémisphère nord, le changement d'albédo contribue au réchauffement du continent. Dans la région affectée par la mousson africaine, l'intensification du phénomène de mousson favorise la migration vers le nord de la transition entre le désert et la steppe. L'albédo moins important de la steppe permet un réchauffement continental plus précoce au printemps et la mise en place de la mousson (Fig. 2(b)). Durant la période de mousson, les plantes recyclent plus efficacement que le sol nu l'eau du sol, renforçant ainsi localement la mousson par recyclage local et relâchement de chaleur latente dans l'atmosphère au moment de la condensation. Lorsque l'océan et la végétation sont tous deux interactifs, le changement de mousson est bien plus intense que la somme des rétroactions individuelles. Le changement de précipitation résultant (Fig. 2(b)) est la manifestation de la forte synergie entre les différentes rétroactions.

\section{Vers l'étude des changements de variabilité interannuelle}

Les simulations couplées océan-atmosphère-glace de mer permettent d' analyser, non seulement les changements de cycle saisonnier moyen, mais aussi les changements de variabilité interannuelle à séculaire, suivant la longueur des simulations. Les premières analyses de changement à partir de simulations couplées GCM ont concerné phénomène ENSO, qui est la manifestation principale du couplage entre l'océan et l'atmosphère à l'échelle interannuelle dans les tropiques [23]. Ces analyses montrent que les caractéristiques d'ENSO au centre du Pacifique sont peu affectées par le changement d'insolation à l'Holocène moyen, mais que la zone d'influence est plus réduite. D'autres études donnent des résultats en ce sens. La Fig. 3 compare le changement de variabilité interannuelle en été, simulé par trois modèles couplés, pour l'Holocène moyen. Cette figure illustre la grande diversité des résultats, en particulier pour Europe, l'Amérique du Nord, l'océan Atlantique nord et les moyennes latitudes de l'océan Pacifique. On peut noter, cependant, que les trois modèles indiquent une augmentation de la variabilité interannuelle des tem- 

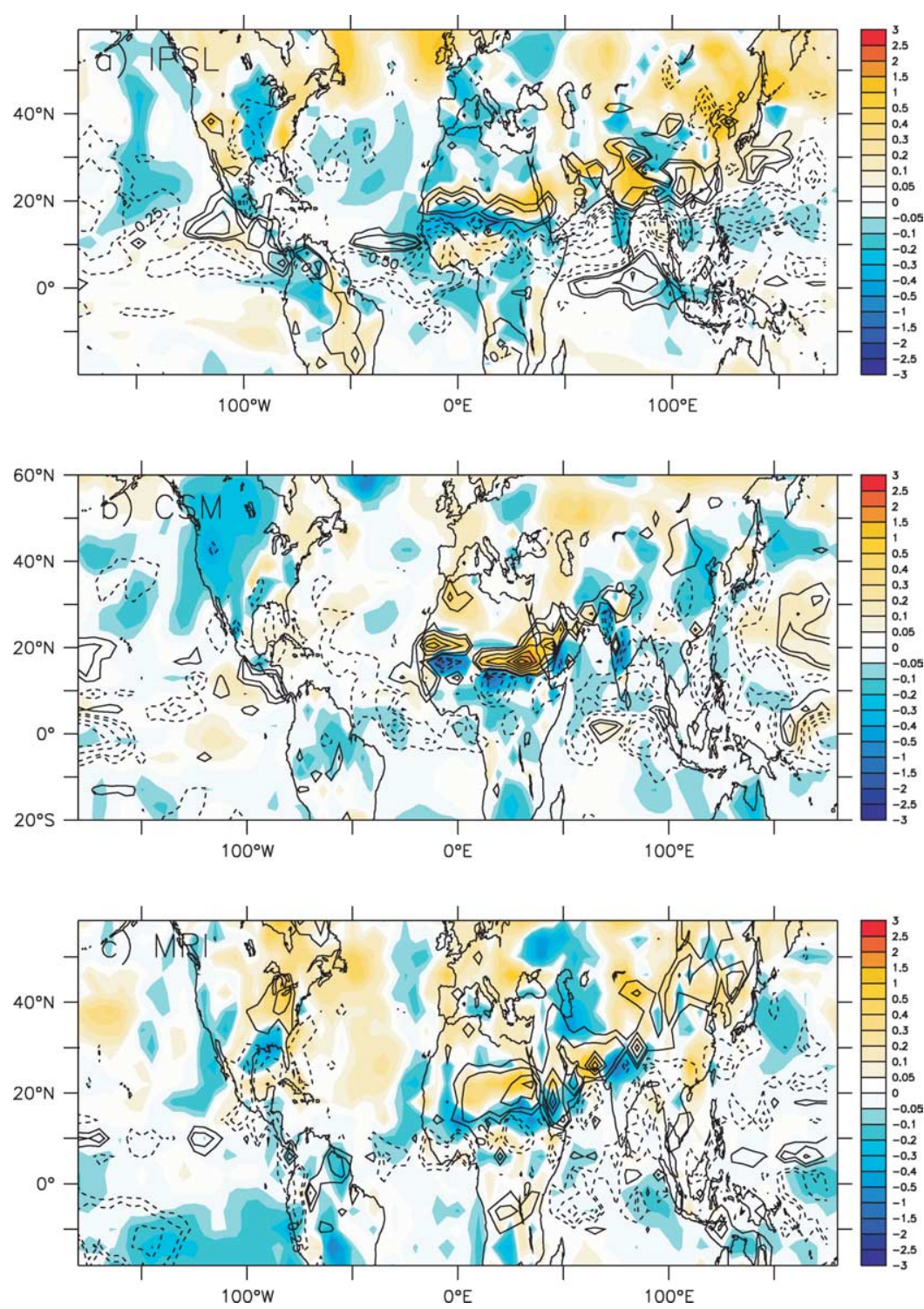

Fig. 3. Changement de variabilité interannuelle (écart type) en été simulé par trois modèles couplés pour le climat l'Holocène moyen. Les couleurs correspondent à la température et les isolignes aux précipitations. Dans les deux cas, la ligne 0 est omise. Pour les températures, les isolignes sont portées tous les $0,1^{\circ} \mathrm{C}$ entre $-0,5$ et $0,5^{\circ} \mathrm{C}$ et tous les $0,5^{\circ} \mathrm{C}$ au-delà. Pour les précipitations, les isolignes sont portées tous les $0,25 \mathrm{~mm} \mathrm{j}^{-1}$ entre $-0,75$ et $0,75 \mathrm{~mm} \mathrm{j}^{-1}$ et tous les $1 \mathrm{~mm} \mathrm{j}^{-1}$ au-delà.

Fig. 3. Change in summer interannual variability (standard deviation) as simulated by three coupled models for the mid-Holocene. The colors stand for temperature, whereas the isolines stand for precipitation. In both cases the 0 line is omitted. Isolines are plotted every $0.1{ }^{\circ} \mathrm{C}$ between -0.5 and $0.5^{\circ} \mathrm{C}$ then every $0.5^{\circ} \mathrm{C}$ for temperature. Isolines are plotted every $0.25 \mathrm{~mm} \mathrm{day}^{-1}$ between -0.75 and $0.75 \mathrm{~mm} \mathrm{day}^{-1}$ then every $\mathrm{mm}$ day $^{-1}$ for precipitation.

pératures sur le continent asiatique et une réduction de variabilité dans la bande tropicale. Dans cette dernière, les précipitations ont également une variabilité réduite, ce qui concorde avec les résultats des coraux [30]. Les trois simulations indiquent également une migration vers le nord de la zone de forte variabilité interannuelle située dans la partie nord de l'ITCZ, qui correspond à la migration vers le nord des zones affectées 
par la mousson dans la région s'étendant de l'Afrique à l'Inde.

L'analyse de la variabilité interannuelle et la façon dont elle a changé dans le passé constitue une nouvelle étape pour les modèles de climat. Elle devrait nous permettre de mieux évaluer les risques de changement de variabilité ou d'intensité des événements extrêmes pouvant survenir dans le futur sous l'impact de l'activité humaine. Elle demande aussi de pouvoir évaluer les impacts relatifs ou combinés des changements de variabilité ou d'état moyen. Cet article montre comment les couplages entre les différents compartiments du système climatique peuvent amplifier la perturbation initiale et de là, la réponse du cycle annuel moyen. De nombreuses inconnues subsistent sur le rôle de la circulation thermocline de l'océan, des surfaces englacées (glace de mer, calottes de glace) ou de la végétation. Il reste aussi à évaluer comment ces différents éléments affectent la variabilité interannuelle à séculaire et le degré de réalisme nécessaire pour représenter correctement les variations de ces différents phénomènes. La deuxième phase du projet PMIP au niveau international, incluant les résultats des simulations couplées océan-atmosphère-végétation offrira un cadre cohérent à la poursuite des études du dernier maximum glaciaire et de l'Holocène moyen et permettra le synthèse des nouveaux indicateurs climatiques nécessaires pour évaluer les résultats des modèles (http://www-lsce.cea.fr/pmip2/).

\section{Remerciements}

Les figures présentées dans cet article ont été réalisées à partir de la base de données PMIP (http://wwwlsce.cea.fr/pmip/) et les données du groupe de travail sur le modèle couplé issu de PMIP.

\section{Références}

[1] A. Berger, Long-term variations of caloric solar radiation resulting from the earth's orbital elements, Quat. Res. 9 (1978) 139-167.

[2] P. Braconnot, S. Joussaume, O. Marti, N. de Noblet, Synergistic feedbacks from ocean and vegetation on the African monsoon response to mid-Holocene insolation, Geophys. Res. Lett. 26 (1999) 2481-2484.

[3] P. Braconnot, M.-F. Loutre, B. Dong, S. Joussaume, P. Valdes, How the simulated change in monsoon at $6 \mathrm{ka} \mathrm{BP}$ is related to the simulation of the modern climate: results from the Paleoclimate Modeling Intercomparison Project, Clim. Dyn. 19 (2002) 107-121.

[4] P. Braconnot, O. Marti, S. Joussaume, Y. Leclainche, Ocean feedback in response to 6 kyr BP insolation, J. Clim. 13 (2000) 1537-1553.

[5] M. Claussen, V. Gayler, The greening of the Sahara during the mid-Holocene: results of an interactive atmosphere-biome model, Global Ecol. Biogeogr. Lett. 6 (1997) 369-377.

[6] CLIMAP, Seasonal reconstructions of the Earth's surface at the last glacial maximum, Map Series Technical Report MC-36, 1981.

[7] R. Doherty, J. Kutzbach, J. Foley, D. Pollard, Fully coupled climate/dynamical vegetation model simulations over Northern Africa during the mid-Holocene, Clim. Dyn. 16 (2000) 561573.

[8] J.-C. Duplessy, N.J. Shackleton, R. Fairbanks, L. Labeyrie, D. Oppo, N. Kallel, Deep water source variation during the last climatic cycle and their impact on th global deep water circulation, Paleoceanography 3 (1988) 343-360.

[9] I. Farrera, S.P. Harrison, I.C. Prentice, G. Ramstein, J. Guiot, P.J. Bartlein, R. Bonnefille, M. Bush, W. Cramer, U. von Grafenstein, K. Holmgren, H. Hooghiemstra, G. Hope, D. Jolly, S.-E. Lauritzen, Y. Ono, S. Pinot, M. Stute, G. Yu, Tropical climates at the Last Glacial Maximum: a new synthesis of terrestrial palaeoclimate data. I. Vegetation, lake-levels and geochemistry, Clim. Dyn. 15 (1999) 823-856.

[10] S.P. Harrison, D. Jolly, F. Laarif, A. Abe-Ouchi, B. Dong, K. Herterich, C. Hewitt, S. Joussaume, J.E. Kutzbach, J. Mitchell, N. de Noblet, P. Valdes, Intercomparison of simulated global vegetation distributions in response to $6 \mathrm{kyr}$ BP orbital forcing, J. Clim. 11 (1998) 2721-2742.

[11] C.D. Hewitt, A.J. Broccoli, J.F.B. Mitchell, R.J. Stouffer, A coupled model study of the last glacial maximum: was part of the North Atlantic relatively warm?, Geophys. Res. Lett. 28 (2001) 1571-1574.

[12] C.D. Hewitt, J.F.B. Mitchell, A fully coupled GCM simulation of the climate of the mid-Holocene, Geophys. Res. Lett. 25 (1998) 361-364.

[13] C.D. Hewitt, R.J. Stouffer, A.J. Broccoli, J.F.B. Mitchell, P.J. Valdes, The effect of ocean dynamics in a coupled GCM simulation of the Last Glacial Maximum, Clim. Dyn. 20 (2003) 203-218.

[14] IPCC, Climate Change 2001, The Scientific Basis, Cambridge University Press, Cambridge, 2001, 98 p.

[15] S. Joussaume, K.E. Taylor, Status of the paleoclimate modeling intercomparison project, in: Proc. 1st Int. AMIP Sci. Conf., WCRP-92, Monterey, USA, 1995, pp. 25-430.

[16] S. Joussaume, K.E. Taylor, P. Braconnot, J.F.B. Mitchell, J.E. Kutzbach, S.P. Harrison, I.C. Prentice, A.J. Broccoli, A. Abe-Ouchi, P.J. Bartlein, C. Bonfils, B. Dong, J. Guiot, K. Herterich, C.D. Hewitt, D. Jolly, J.W. Kim, A. Kislov, A. Kitoh, M.-F. Loutre, V. Masson, B. McAvaney, N. McFarlane, N. de Noblet, W.R. Peltier, J.Y. Peterschmitt, D. Pollard, D. Rind, J.-F. Royer, M.E. Schlesinger, J. Syktus, S. Thompson, P. Valdes, G. Vettoretti, R.S. Webb, U. Wyputta, Monsoon changes for 6000 years ago: results of 18 simulations from the 
Paleoclimate Modeling Intercomparison Project (PMIP), Geophys. Res. Lett. 26 (1999) 859-862.

[17] M. Kageyama, P.J. Valdes, G. Ramstein, C. Hewitt, U. Wyputta, Northern hemisphere storm tracks in present day and Last Glacial Maximum climate simulations: a comparison of the European PMIP models, J. Clim. 12 (1999) 742-760.

[18] M. Kageyama, O. Peyron, S. Pinot, P. Tarasov, J. Guiot, S. Joussaume, G. Ramstein, The Last Glacial Maximum climate over Europe and western Siberia: a PMIP comparison between models and data, Clim. Dyn. 17 (2001) 23-43.

[19] S.J. Kim, G.M. Flato, G.J. Boer, A coupled climate model simulation of the Last Glacial Maximum, Part 2: approach to equilibrium, Clim. Dyn. 20 (2003) 635-661.

[20] A. Kitoh, S. Murakami, H. Koide, A simulation of the Last Glacial Maximum with a coupled atmosphere-ocean GCM, Geophys. Res. Lett. 28 (2001) 2221-2224.

[21] N. Koc Karpuz, H. Schrader, Surface sediment distribution and Holocene paleotemperature variations in the Greenland, Iceland and Norwegian Sea, Paleoceanography 5 (1990) 557580.

[22] J.E. Kutzbach, Z. Liu, Response of the African monsoon to orbital forcing and ocean feedbacks in the Middle Holocene, Science 278 (1997) 440-443.

[23] B.L. Otto-Bliesner, El Niño/La niña and Sahel precipitation during the Middle Holocene, Geophys. Res. Lett. 26 (1999) 87-90.

[24] W.R. Peltier, Ice age paleotopography, Science 265 (1994) 195-201.

[25] S. Pinot, G. Ramstein, S.P. Harrison, I.C. Prentice, J. Guiot, M. Stute, S. Joussaume, Tropical paleoclimates at the Last
Glacial Maximum: comparison of Paleoclimate Modeling Intercomparison Project (PMIP) simulations and paleodata, Clim. Dyn. 15 (1999) 857-874.

[26] D. Raynaud, J. Jouzel, J.-M. Barnola, J. Chappelaz, R. Delmas, C. Lorius, The ice record of greenhouse gases, Science 259 (1993) 926-934.

[27] W.F. Ruddiman, A.C. Mix, The north and equatorial Atlantic at 9000 and $6000 \mathrm{yr}$ BP, in: H.E.J. Wright, J.E. Kutzbach, T. Webb III, W.F. Ruddiman, F.A. Street-Perrott, P.J. Bartlein (Eds.), Global Climates Since the Last Glacial Maximum, University of Minnesota Press, 1993, pp. 94-124.

[28] S.I. Shin, Z. Liu, B. Otto-Bliesner, E.C. Brady, J.E. Kutzbach, S.P. Harrison, A simulation of the Last Glacial Maximum climate using the NCAR-CCSM, Clim. Dyn. 20 (2003) $127-$ 151.

[29] D. Texier, N. de Noblet, S.P. Harrison, A. Haxeltine, D. Jolly, S. Joussaume, F. Laarif, I.C. Prentice, P. Tarasov, Quantifying the role of biosphere-atmosphere feedbacks in climate change: coupled model simulations for 6000 years BP and comparison with palaeodata for northern Eurasia and northern Africa, Clim. Dyn. 13 (1997) 865-882.

[30] A.W. Tudhope, C.P. Chilcott, M.T. McCulloch, E.R. Cook, J. Chappell, R.M. Ellam, D.W. Lea, J.M. Lough, G.B. Shimmield, Variability in the El Nino southern oscillation through a glacial-interglacial cycle, Science 291 (2001) 1511-1517.

[31] U. Wyputta, B.J. McAvaney, Influence of vegetation changes during the Last Glacial Maximum using the BMRC atmospheric general circulation model, Clim. Dyn. 17 (2001) 923-932. 